Provided for non-commercial research and education use. Not for reproduction, distribution or commercial use.

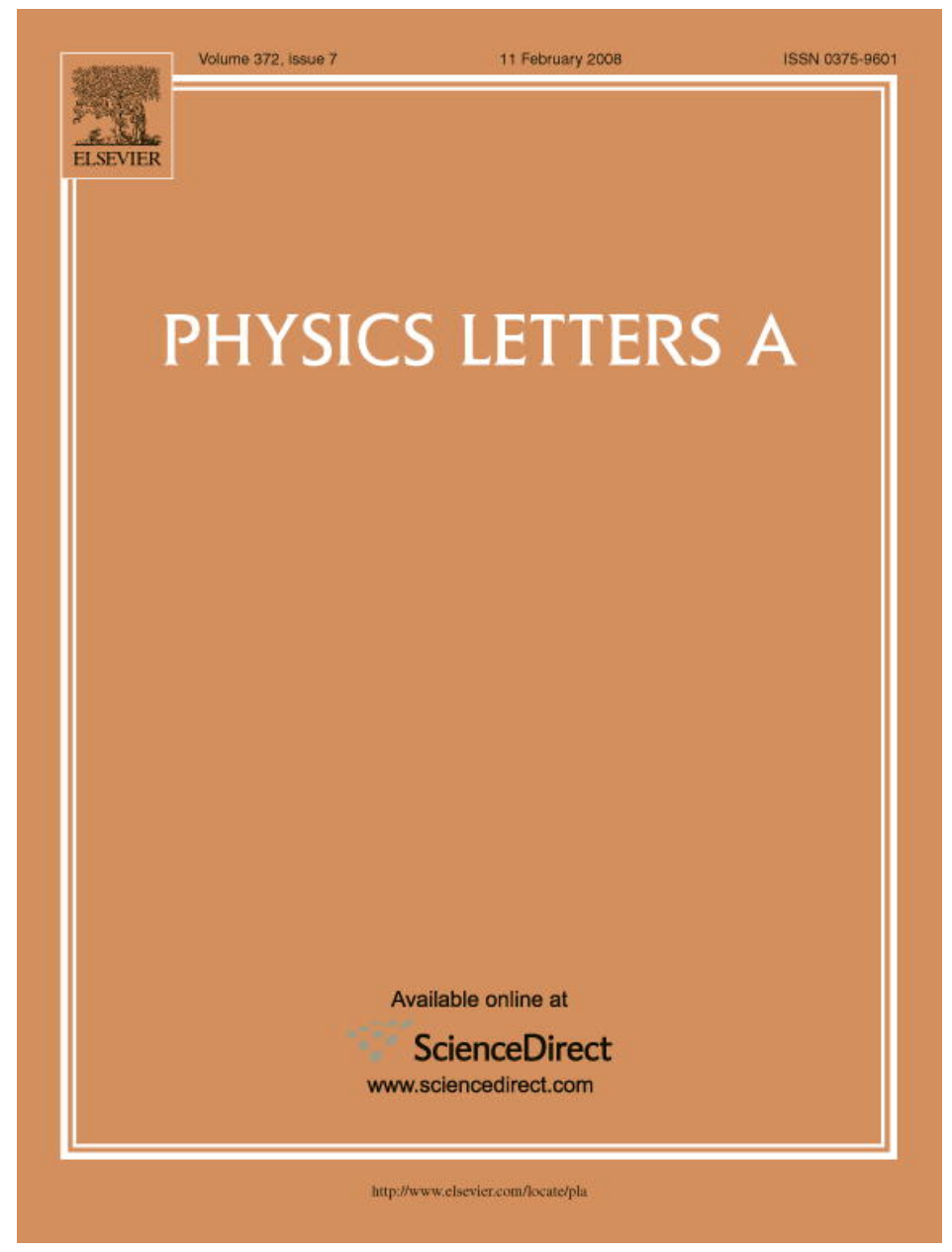

This article was published in an Elsevier journal. The attached copy

is furnished to the author for non-commercial research and education use, including for instruction at the author's institution, sharing with colleagues and providing to institution administration.

Other uses, including reproduction and distribution, or selling or licensing copies, or posting to personal, institutional or third party websites are prohibited.

In most cases authors are permitted to post their version of the article (e.g. in Word or Tex form) to their personal website or institutional repository. Authors requiring further information regarding Elsevier's archiving and manuscript policies are encouraged to visit:

http://www.elsevier.com/copyright 


\title{
On the relation between the fractional Brownian motion and the fractional derivatives
}

\author{
Manuel Duarte Ortigueira *, Arnaldo Guimarães Batista \\ UNINOVA/DEE, Campus da FCT da UNL, Quinta da Torre, 2825-114 Monte da Caparica, Portugal \\ Received 5 January 2007; received in revised form 23 July 2007; accepted 22 August 2007 \\ Available online 8 September 2007 \\ Communicated by A.P. Fordy
}

\begin{abstract}
The definition and simulation of fractional Brownian motion are considered from the point of view of a set of coherent fractional derivative definitions. To do it, two sets of fractional derivatives are considered: (a) the forward and backward and (b) the central derivatives, together with two representations: generalised difference and integral. It is shown that for these derivatives the corresponding autocorrelation functions have the same representations. The obtained results are used to define a fractional noise and, from it, the fractional Brownian motion. This is studied. The simulation problem is also considered.
\end{abstract}

(c) 2007 Elsevier B.V. All rights reserved.

Keywords: Forward and backward fractional derivatives; Generalised Cauchy derivative; Liouville derivative; Differintegration; Central fractional derivatives;

Fractional stochastic process; Fractional Brownian motion

\section{Introduction}

Fractional Brownian motion (fBm) and $1 / f$ noises are well-known designations for some kinds of stochastic processes with fractional characteristics that are very frequent in Nature and in daily applications [1-4]. In parallel, self-similarity and long range dependence are interconnected notions and appear in a variety of contexts [1-4]. However, it is not clear how we can establish a bridge between these notions and several definitions of fractional derivative. Here we will try to present a new step into that goal.

Fractional Brownian motion was introduced first by Kolmogorov [5]. Later, Mandelbrot and Van Ness [1,2] proposed it as a model for nonstationary signals, with stationary increments, that are useful in understanding phenomena with long range dependence and with a frequency dependence of the form $1 / f^{\alpha}$, with $\alpha$ non-integer. The proposal of Mandelbrot and Van Ness can be stated as follows.

Let $H, 0<H<1$, be the called Hurst parameter and $b_{0}$ a number. Then the $\mathrm{fBm}, B_{H}(t)$, with parameter $H$ is defined by:

$$
B_{H}(t)-B_{H}(0)=\frac{1}{\Gamma(H+1 / 2)}\left\{\int_{-\infty}^{0}\left[(t-\tau)^{H-1 / 2}-(-\tau)^{H-1 / 2}\right] d B(\tau)+\int_{0}^{t}(t-\tau)^{H-1 / 2} d B(\tau)\right\},
$$

where $B_{H}(0)=b_{0}$, and $B(t)$ is the standard Brownian motion. Following a common practice in engineering texts we will introduce the stationary white noise process, $w(t)$, instead of $d B(t)$. Although there are other definitions and approaches to fBm, the above definition is usually accepted.

In this Letter, we will give a new format to (1) that leads to a formulation proposed before $[4,6,7]$ that represents the fBm as an integral of a fractional noise. This is obtained from the fractional differintegration of white noise. The use of different derivatives

\footnotetext{
* Corresponding author. Tel.: +35112948520; fax: +35112948532.

E-mail addresses: mdo@dee.fct.unl.pt (M.D. Ortigueira), agb@fct.unl.pt (A.G. Batista).
} 
allows us to obtain different fractional noises but with the same autocorrelation function. We exploit this fact to propose a new way of simulating the fBm [7]. We describe several definitions of fractional derivatives that are coherently interconnected. In particular, the Grünwald-Letnikov derivatives and similar can be used [8-11].

The Letter outline is as follows. In Section 2, we establish a new relation between $\mathrm{fBm}$ and the fractional derivatives. These are presented in Section 3. We study two types of fractional derivatives: causal and central and two formulations: generalised incremental ratio and convolutional. They are used in Section 4 to compute the autocorrelation function of the derivative of stationary stochastic processes. The proposed approach for defining $\mathrm{fBm}$ is presented in the following section, where we will study its main features. The simulation problem is also considered from the point of view of the proposed theory.

\section{A new look at Mandelbrot and Van Ness definition}

Let us return to (1). Rewrite it in the format:

$$
\begin{aligned}
& \frac{1}{\Gamma(H+1 / 2)}\left\{\int_{-\infty}^{0} w(\tau)\left[(t-\tau)^{H-1 / 2}-(-\tau)^{H-1 / 2}\right] d \tau+\int_{0}^{t} w(\tau)(t-\tau)^{H-1 / 2} d \tau\right\} \\
& =\frac{1}{\Gamma(H+1 / 2)}\left\{\int_{-\infty}^{+\infty} w(\tau)\left[(t-\tau)^{H-1 / 2} u(t-\tau)-(-\tau)^{H-1 / 2} u(-\tau)\right]\right\} d \tau,
\end{aligned}
$$

where $u(t)$ is the Heaviside unit step. The inner expression can be considered as the application of the Barrow formula to the function $(t-\tau)^{H-1 / 2} u(t-\tau)$. By a straightforward computation, we have:

$$
\frac{1}{\Gamma(H-1 / 2)} \int_{0}^{t}(s-\tau)^{H-3 / 2} u(s-\tau) d s=\frac{1}{\Gamma(H+1 / 2)}\left[(t-\tau)^{H-1 / 2} u(t-\tau)-(-\tau)^{H-1 / 2} u(-\tau)\right] .
$$

With this, we have:

$$
\begin{aligned}
B_{H}(t)-B_{H}(0) & =\frac{1}{\Gamma(H-1 / 2)} \int_{-\infty}^{+\infty} w(\tau) \int_{0}^{t}(s-\tau)^{H-3 / 2} u(s-\tau) d s d \tau \\
& =\frac{1}{\Gamma(H-1 / 2)} \int_{0}^{t} \int_{-\infty}^{s} w(\tau)(s-\tau)^{H-3 / 2} d \tau d s .
\end{aligned}
$$

The inner integral is the Liouville forward derivative of order $-H+1 / 2$ [8]:

$$
D_{+}^{\alpha} w(t)=\frac{1}{\Gamma(H-1 / 2)} \int_{-\infty}^{t} w(\tau)(t-\tau)^{H-3 / 2} d \tau
$$

This means that

$$
B_{H}(t)-B_{H}(0)=\int_{0}^{t} D_{+}^{\alpha} w(\tau) d \tau
$$

where $\alpha=-H+1 / 2$ is the derivative order. As $H \in(0,1), \alpha \in(-1 / 2,1 / 2)$. The expression (4) is similar to the corresponding definition of Brownian motion, provided that $\alpha=0(H=1 / 2)$. This formula was proposed in [4,6] and it is interesting, since it suggests us the possible use of other fractional derivative definitions alternative to the Liouville definition. We must remark also that it is valid for non-Gaussian white noises.

\section{The fractional derivative definitions}

\subsection{On the fractional derivatives}

Fractional calculus is a 300 years young field that has been rediscovered by scientists and engineers and applied in an increasing number of fields. Motivated by the excellent textbooks [12,13], the increase in the number of physical and engineering processes using fractional derivatives is a fact. However, similar applications based in different definitions lead to different results. Riemann-Liouville, Caputo, Grünwald-Letnikov, Hadamard, Marchaud, are some of the known definitions [8,12,13]. From 
a purely mathematical point of view it is legitimate to accept and even use one or all, but from signal processing point of view, we should accept only the definitions that might lead to a fractional systems theory coherent with the usual practice, and accepted notions and concepts such as the impulse response and transfer function. In previous papers [8-11] we made some contributions towards this goal on proposing coherent approaches to fractional derivative definitions. Here, we present these definitions in a very concise way. We will describe two types of fractional derivatives: causal and central and two formulations: generalised incremental ratio and convolutional.

\subsection{The forward and backward derivatives}

Let $f(z)$ be a complex variable function analytic in a region that includes a half straight line starting at $z$ and defined by $z-n h$, with $n \in Z ; h$ is any complex in the right-hand d'Argand plane. Consider the $U$-shaped contours represented in Fig. 1.

Assume that this line is inside the analyticity region.

Definition 1. We define the forward and backward Grünwald-Letnikov fractional $\alpha$ order derivatives by the left-hand sides in (5) and (6) below.

With these definitions and under the above conditions we can state the following interesting result $[8,9]$ :

\section{Theorem 1.}

$$
D_{+}^{\alpha} f(z)=\lim _{h \rightarrow 0+} \frac{\sum_{k=0}^{\infty}(-1)^{k}\left(\begin{array}{l}
\alpha \\
k
\end{array}\right) f(z-k h)}{h^{\alpha}}=\frac{\Gamma(\alpha+1)}{2 \pi i} \oint_{C_{f}} f(s) \frac{1}{(s-z)^{\alpha+1}} d s .
$$

The right-hand side is the generalised Cauchy derivative. $C_{f}$ is the left-hand $U$-shaped path in Fig. 1.

Making a substitution $h \rightarrow-h$ we obtain the backward Grünwald-Letnikov derivative on the left and a generalised Cauchy with a right-hand branch cut line:

$$
D_{-}^{\alpha} f(z)=\lim _{h \rightarrow 0+}(-1)^{\alpha} \frac{\sum_{k=0}^{\infty}(-1)^{k}\left(\begin{array}{l}
\alpha \\
k
\end{array}\right) f(z+k h)}{h^{\alpha}}=\frac{\Gamma(\alpha+1)}{2 \pi i} \oint_{C_{b}} f(s) \frac{1}{(s-z)^{\alpha+1}} d s .
$$

We must remark that the right-hand side remains the same except for the integration path. Now, it lies in the right-hand complex plane defined by a vertical straight line passing over $z$.

We can go a bit further by deforming the contour used in (5) and (6) in order to transform it in the Hankel path [7]. We obtain:

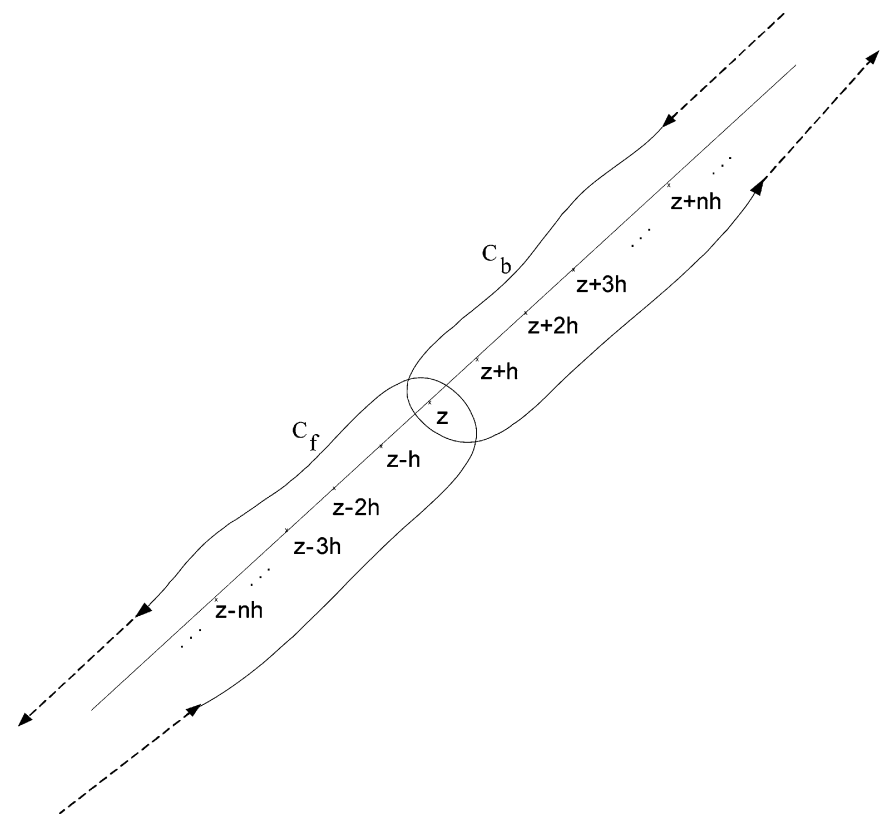

Fig. 1. $U$-shaped contours for forward and backward derivatives. 


\section{Theorem 2.}

$$
D_{ \pm}^{\alpha} f(z)=\frac{e^{j(\pi-\theta) \alpha}}{\Gamma(-\alpha)} \int_{0}^{\infty} \frac{\left[f\left(x \cdot e^{j \theta}+z\right)-\sum_{0}^{N} \frac{f^{(n)}(z)}{n !} e^{j n \theta} x^{n}\right]}{x^{\alpha+1}} d x
$$

where $\theta$ is the angle between the positive real axis and the branch cut line. This is a Hadamard like regularised integral [16], but obtained without rejecting any infinite part. If $\theta=\pi(+)$, we have the forward derivative, while with $\theta=0(-)$, we obtain the backward one.

Corollary 1. If $f(z)$ is a Laplace transformable function, it can be shown that [8]

$$
D_{+}^{\alpha} f(z)=\lim _{h \rightarrow 0+} \frac{\sum_{k=0}^{\infty}(-1)^{k}\left(\begin{array}{l}
\alpha \\
k
\end{array}\right) f(z-k h)}{h^{\alpha}}=\frac{1}{\Gamma(-\alpha)} \int_{0}^{\infty} f(z-\tau) \tau^{-\alpha-1} d \tau
$$

and

$$
D_{-}^{\alpha} f(z)=\lim _{h \rightarrow 0+}(-1)^{\alpha} \frac{\sum_{k=0}^{\infty}(-1)^{k}\left(\begin{array}{l}
\alpha \\
k
\end{array}\right) f(z+k h)}{h^{\alpha}}=\frac{(-1)^{-\alpha}}{\Gamma(-\alpha)} \int_{0}^{\infty} f(z+\tau) \tau^{-\alpha-1} d \tau,
$$

where the right-hand sides in (8) and (9) are the forward and backward Liouville derivatives. They correspond to the output of a fractional differintegrator [8]. In the following, we shall be working with the forward derivative.

\subsection{The central derivatives}

The central fractional derivatives were proposed, for the first time, in [10,11]. Assume that $\alpha>-1$ and $h \in R^{+}$(only by simplicity).

Definition 2. We define the types 1 and 2 fractional central derivatives, respectively by:

$$
D_{c_{1}}^{\alpha} f(z)=\lim _{h \rightarrow 0} \frac{\Gamma(\alpha+1)}{h^{\alpha}} \sum_{k=-\infty}^{+\infty} \frac{(-1)^{k}}{\Gamma(\alpha / 2-k+1) \Gamma(\alpha / 2+k+1)} f(z-k h)
$$

and

$$
D_{c_{2}}^{\alpha} f(z)=\lim _{h \rightarrow 0} \frac{\Gamma(\alpha+1)}{h^{\alpha}} \sum_{k=-\infty}^{+\infty} \frac{(-1)^{k}}{\Gamma[(\alpha+1) / 2-k+1] \Gamma[(\alpha-1) / 2+k+1]} f(z-k h+h / 2) .
$$

Theorem 3. In the above conditions, the integral formulation for the type 1 derivative is

$$
D_{c_{1}}^{\alpha} f(z)=\frac{\Gamma(\alpha+1)}{2 \pi i} \int_{C} f(z+s) \frac{1}{(s)_{l}^{\alpha / 2+1}(-s)_{r}^{\alpha / 2}} d s
$$

while for the type 2 derivative is

$$
D_{c_{2}}^{\alpha} f(z)=\frac{\Gamma(\alpha+1)}{2 \pi i} \int_{C} f(z+s) \frac{1}{(s)_{l}^{(\alpha+1) / 2}(-s)_{r}^{(\alpha+1) / 2}} d s,
$$

where $C$ is a two straight lines integration path (see Fig. 2). The subscripts " $l$ " and " $r$ " mean respectively that the power functions have the left and right half real axes as branch cut lines. These integrals represent again generalisations of the Cauchy derivative.

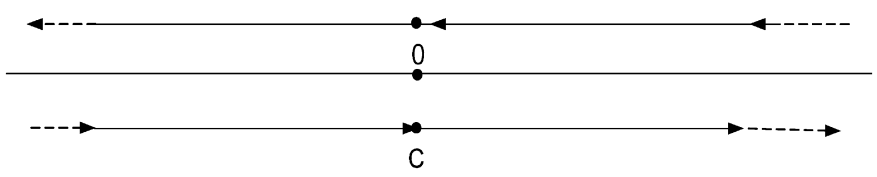

Fig. 2. Segments for the computation of the integrals (12) and (13). 
Corollary 2. Computing the integrals using the above paths, we obtain for the type 1 case [10,11]

$$
\lim _{h \rightarrow 0} \frac{\Gamma(\alpha+1)}{h^{\alpha}} \sum_{k=-\infty}^{+\infty} \frac{(-1)^{k}}{\Gamma(\alpha / 2-k+1) \Gamma(\alpha / 2+k+1)} f(z-k h)=\frac{1}{2 \Gamma(-\alpha) \cos (\alpha \pi / 2)} \int_{-\infty}^{\infty} f(z-x) \frac{1}{|x|^{\alpha+1}} d x
$$

and for the type 2 case:

$$
\begin{aligned}
& \lim _{h \rightarrow 0} \frac{\Gamma(\alpha+1)}{h^{\alpha}} \sum_{k=-\infty}^{+\infty} \frac{(-1)^{k}}{\Gamma[(\alpha+1) / 2-k+1] \Gamma[(\alpha-1) / 2+k+1]} f(z-k h+h / 2) \\
& \quad=-\frac{1}{2 \Gamma(-\alpha) \sin (\alpha \pi / 2)} \int_{-\infty}^{\infty} f(z-x) \frac{\operatorname{sgn}(x)}{|x|^{\alpha+1}} d x .
\end{aligned}
$$

The right-hand sides in (14) and (15) are the so-called Riesz and modified Riesz potentials [13]. They supply us with two integral formulations for the central derivatives that can be useful in the simulation of fractional noises.

\section{Derivatives of stationary stochastic processes}

Assume that $f(t)$ is a stationary stochastic process with $R_{f}(t)$ as its autocorrelation function. We will assume also that $f(t)$ has a zero mean. If this is not the case, we may have problems when $\alpha$ is negative, since the corresponding derivatives will be divergent; when $\alpha$ is positive the derivative will be zero, for all the definitions. To see this, we can use, for example, the left-hand side in (8). Insert $f(z)=1$ there. We obtain for the numerator $\sum_{k=0}^{\infty}(-1)^{k}\left(\begin{array}{l}\alpha \\ k\end{array}\right)$. As it is well known [14]:

$$
(1-a)^{\alpha}=\sum_{k=0}^{\infty}(-1)^{k}\left(\begin{array}{l}
\alpha \\
k
\end{array}\right) a^{k}
$$

when $a=1$, we conclude that:

$$
\sum_{k=0}^{\infty}(-1)^{k}\left(\begin{array}{l}
\alpha \\
k
\end{array}\right)= \begin{cases}0, & \alpha>0 \\
\infty, & \alpha<0\end{cases}
$$

where $\alpha$ is not a negative integer.

In agreement with the results in the previous section and, at least conceptually, we can use all the above formulae for defining the fractional derivative of a stationary stochastic process. Without any special difficulty and only with some mathematical work, we can show that the autocorrelations obtained with the Grünwald-Letnikov derivatives (the central analogues) are equal to those obtained with the Liouville (Riesz) integrals. On the other hand, from the autocorrelation point of view, there is no difference between the forward (central type 1) and backward (central type 2) derivatives. So and to go a bit further, we will compute the autocorrelation function of the stochastic processes obtained by the Grünwald-Letnikov derivatives and the central analogues-(5) and (10).

\subsection{Forward case}

From (5), we can conclude that:

$$
R_{f}^{\alpha}\left(t_{1}, t_{2}\right)=\lim _{h \rightarrow 0+} \frac{\sum_{k=0}^{\infty} \sum_{n=0}^{\infty}\left(\begin{array}{l}
\alpha \\
k
\end{array}\right)(-1)^{k-n}\left(\begin{array}{l}
\alpha \\
n
\end{array}\right) R_{f}\left[t_{1}-t_{2}-(k-n) h\right]}{h^{2 \alpha}} .
$$

With a change in the summation variable, it is not hard to show that, if $\alpha>-1 / 2,[15]$ :

$$
R_{f}^{\alpha}\left(t_{1}, t_{2}\right)=\lim _{h \rightarrow 0} \frac{\Gamma(2 \alpha+1)}{h^{2 \alpha}} \sum_{k=-\infty}^{+\infty} \frac{(-1)^{k}}{\Gamma(\alpha-k+1) \Gamma(\alpha+k+1)} R_{f}\left(t_{1}-t_{2}-k h\right)
$$

So, the autocorrelation function of the forward $\alpha$-order derivative of a stationary stochastic process is the type 1 centred derivative of order $2 \alpha$, provided that $\alpha>-1 / 2$. This means that the forward fractional derivative of order $\leqslant-1 / 2$ of a stationary stochastic process is not stationary or may not exist. 


\subsection{Central case}

For this case, we proceed as above, but using the type 1 derivative (10). We have then:

$$
R_{f}^{\alpha}\left(t_{1}, t_{2}\right)=\lim _{h \rightarrow 0} \frac{\Gamma(2 \alpha+1)}{h^{2 \alpha}} \sum_{k=-\infty}^{+\infty} \sum_{n=-\infty}^{+\infty} \frac{(-1)^{k+n} R_{f}\left[t_{1}-t_{2}-(k-n) h\right]}{\Gamma(\alpha / 2-k+1) \Gamma(\alpha / 2+k+1) \Gamma(\alpha / 2-n+1) \Gamma(\alpha / 2+n+1)} .
$$

We can simplify this expression with a change in the summation variable in one series, and using the following relation [16]:

$$
\sum_{k=-\infty}^{+\infty} \frac{1}{\Gamma(a-k+1) \Gamma(b-k+1) \Gamma(c+k+1) \Gamma(d+k+1)}=\frac{\Gamma(a+b+c+d+1)}{\Gamma(a+c+1) \Gamma(b+c+1) \Gamma(a+d+1) \Gamma(b+d+1)} .
$$

With it, it is not difficult to show that:

$$
R_{f}^{\alpha}\left(t_{1}, t_{2}\right)=\lim _{h \rightarrow 0} \frac{\Gamma(2 \alpha+1)}{h^{2 \alpha}} \sum_{k=-\infty}^{+\infty} \frac{(-1)^{k}}{\Gamma(\alpha-k+1) \Gamma(\alpha+k+1)} R_{f}\left(t_{1}-t_{2}-k h\right)
$$

that is equal to (17).

From the above results we conclude that, from the autocorrelation point of view, all the definitions lead to the same result. This conclusion was probably expected, since for a stationary stochastic process, there is no privileged direction of time flow.

These considerations mean that it seems to be indifferent to use one or other derivative. The problem at hand can give us some insight into the definition we should adopt. In problems involving time as independent variable we must use (2) because of its causal character. When causality is not involved we must use a central derivative.

\subsection{Some computational issues}

Looking at (17) and (20) we note that the right-hand side is function of $t_{1}-t_{2}$ only. So, we can write:

$$
R_{f}^{\alpha}(t)=\lim _{h \rightarrow 0} \frac{\Gamma(2 \alpha+1)}{h^{2 \alpha}} \sum_{k=-\infty}^{+\infty} \frac{(-1)^{k}}{\Gamma(\alpha-k+1) \Gamma(\alpha+k+1)} R_{f}(t-k h) .
$$

According to the central type 1 derivative definition and, as we referred before,

$$
R_{f}^{\alpha}(t)=D_{c_{1}}^{2 \alpha} R_{f}(t)
$$

and

$$
R_{f}^{\alpha}(t)=\frac{1}{2 \Gamma(-2 \alpha) \cos (\alpha \pi)} \int_{-\infty}^{\infty} R_{f}(t-x) \frac{1}{|x|^{2 \alpha+1}} d x
$$

concluding that the autocorrelation function of the $\alpha$-order derivative of a stationary stochastic process is the $2 \alpha$-order central derivative of the autocorrelation of the process. Care must be taken, because the process may not be stationary. In Section 5.1, we will return to this subject.

In practical applications, we may need to compute a derivative of a signal for which a closed form is not available and we are obliged to truncate the summation or the integral. This leads to an error. This problem was studied in [11,12] in connection with the so-called there "short-memory" principle. Here, we will obtain a similar result for the autocorrelation case. Consider the truncation of the integral in (23) and assume that the autocorrelation of the original process $f(t)$ is a bounded function- $-\left|R_{f}(t)\right|<M-\mathrm{known}$ inside an interval that we will assume to be symmetric relatively to the origin, $[-L ; L]$, only by simplicity. We conclude that the error is bounded:

$$
E<\frac{M}{|\Gamma(-2 \alpha) \cos (\alpha \pi)|} \int_{L}^{\infty} \frac{1}{x^{2 \alpha+1}} d x=\frac{M L^{-2 \alpha}}{|\Gamma(-2 \alpha) \cos (\alpha \pi)|}=\frac{|\Gamma(2 \alpha+1)|}{\pi} M L^{-2 \alpha} .
$$

This result is identical to the one obtained in [12]. A similar result can be obtained for the summation in (21). However, here we have an error bound that is function of $h$. From the properties of the gamma functions, we obtain easily:

$$
\frac{(-1)^{k}}{\Gamma(\alpha-k+1)}=-\frac{\sin (\alpha \pi)}{\pi} \Gamma(-\alpha+k)
$$


and

$$
\frac{(-1)^{k}}{\Gamma(\alpha-k+1) \Gamma(\alpha+k+1)}=-\frac{\sin (\alpha \pi)}{\pi} \frac{\Gamma(-\alpha+|k|)}{\Gamma(\alpha+|k|+1)} .
$$

As the quotient of the two gamma functions in (26) tends asymptotically enough to $|k|^{-2 \alpha-1}$ when $|k|$ is high [11,13], we obtain

$$
\left|\frac{(-1)^{k}}{\Gamma(\alpha-k+1) \Gamma(\alpha+k+1)}\right| \sim \frac{1}{\pi}|k|^{-2 \alpha-1} .
$$

This leads to an error:

$$
E(h) \sim \frac{|\Gamma(\alpha+1)|}{\pi} \sum_{k=L+1}^{+\infty}|k / h|^{-2 \alpha-1} h
$$

and to (24) again.

\section{Returning to the fractional Brownian motion}

\subsection{Definition and properties}

Now, we are going to use the above results to study the fractional Brownian motion (fBm) that we proposed in (4) [4,6]. Assume now that we are computing the fractional derivative of the white noise, $w(t)$ with power equal to $\sigma^{2}$.

Definition 3. We define a fractional noise by:

$$
r_{\alpha}(t)=D^{\alpha} w(t)
$$

If $w(t)$ is Gaussian, we will call $r_{\alpha}(t)$ fractional Gaussian noise. According to the results in Section $4, D^{\alpha}$ represents any of the defined derivatives.

As known, the autocorrelation function of the white noise is $\sigma^{2} \delta(t)$. Inserting this into (21), we obtain for the derivative autocorrelation:

$$
R_{r}^{\alpha}(t)=\lim _{h \rightarrow 0} \frac{\Gamma(2 \alpha+1)}{h^{2 \alpha}} \sum_{-\infty}^{+\infty} \frac{(-1)^{k}}{\Gamma(\alpha-k+1) \Gamma(\alpha+k+1)} \delta(t-k h) .
$$

The right-hand side is a sequence of weighted impulses that become close together as $h$ goes to zero. From (23) we conclude immediately that:

$$
R_{r}^{\alpha}(t)=\frac{1}{2 \Gamma(-2 \alpha) \cos (\alpha \pi)}|t|^{-2 \alpha-1}
$$

giving an interesting result:

$$
|t|^{-2 \alpha-1}=\frac{\pi}{\sin (-\alpha \pi)} \lim _{h \rightarrow 0} \frac{1}{h^{2 \alpha}} \sum_{-\infty}^{+\infty} \frac{(-1)^{k}}{\Gamma(\alpha-k+1) \Gamma(\alpha+k+1)} \delta(t-k h)
$$

valid for $\alpha>-1 / 2$. Returning back to (31) we can deduce that we must have

$$
\left\{\begin{array}{l}
2 \alpha+1>0, \\
\Gamma(-2 \alpha) \cos (\alpha \pi)>0
\end{array}\right.
$$

to guarantee that (31) represents an autocorrelation function, having a maximum at the origin. The first condition $(\alpha>-1 / 2)$ was already assumed. As

$$
\frac{1}{2 \Gamma(-2 \alpha) \cos (\alpha \pi)}=-\frac{\Gamma(2 \alpha+1) \cdot \sin (2 \alpha \pi)}{2 \pi \cos (\alpha \pi)}=-\frac{\Gamma(2 \alpha+1) \sin (\alpha \pi)}{\pi}
$$

it is not hard to see that for $-1 / 2<\alpha<0$ and $\alpha \in(2 n, 2 n+1), n \in Z^{+}$, we obtain valid autocorrelation functions. We conclude that, in the interval $-1 / 2<\alpha<1 / 2$ we obtain a stationary process in the integration case $(\alpha<0)$ and nonstationary in the derivative case $(\alpha>0)$. This fractional noise will be used next to define the fractional Brownian motion. 
Definition 4. Let $r_{\alpha}(t)$ be a fractional noise. Define a process $v_{\alpha}(t), t \geqslant 0$, by:

$$
v_{\alpha}(t)=\int_{0}^{t} r_{\alpha}(\tau) d \tau
$$

We will call this process a fractional Brownian motion (or generalised Wiener-Lévy process). It is not difficult to show that it enjoys all the properties normally required for the fBm [6]:

(1) $v_{\alpha}(0)=0$ and $E\left\{v_{\alpha}(t)\right\}=0$ for every $t \geqslant 0$. If $w(t)$ is Gaussian, so it is $r_{\alpha}(t)$ and $v_{\alpha}(t)$. The proposed definitions do not need the gaussianity.

(2) The covariance is [16]:

$$
E\left[v_{\alpha}(t) v_{\alpha}(s)\right]=\frac{\sigma^{2}}{2 \Gamma(-2 \alpha+2) \cdot \cos \alpha \pi}\left[|t|^{-2 \alpha+1}+|s|^{-2 \alpha+1}-|t-s|^{-2 \alpha+1}\right]
$$

valid for $|\alpha|<1 / 2$. Putting $H=-\alpha+1 / 2$ with $H \in(0,1)$, we obtain the usual formulation:

$$
E\left[v_{\alpha}(t) v_{\alpha}(s)\right]=\frac{V_{H}}{2}\left[|t|^{2 H}+|s|^{2 H}-|t-s|^{2 H}\right]
$$

with

$$
V_{H}=\frac{\sigma^{2}}{\Gamma(2 H+1) \sin H \pi}
$$

a much more simple expression than the one currently used [1]. The variance is readily obtained:

$$
E\left[v_{\alpha}(t)^{2}\right]=V_{H}|t|^{2 H} \text {. }
$$

(3) The process has stationary increments.

Letting the increments be defined by

$$
\Delta v_{\alpha}(t, s)=v_{\alpha}(t)-v_{\alpha}(s)=\int_{s}^{t} r_{\alpha}(\tau) d \tau
$$

its variance is given by [6]:

$$
\operatorname{Var}\left\{\Delta v_{\alpha}(t, s)\right\}=\sigma^{2} \frac{|t-s|^{-2 \alpha+1}}{2 \Gamma(-2 \alpha+2) \cdot \cos \alpha \pi} \text {. }
$$

(4) The process is self similar.

From (37), we have:

$$
E\left[v_{\alpha}(a t) v_{\alpha}(a s)\right]=\frac{V_{H}}{2}\left[|a . t|^{2 H}+|a . s|^{2 H}-|a . t-a . s|^{2 H}\right]=\frac{V_{H}}{2}|a|^{2 H}\left[|t|^{2 H}+|s|^{2 H}-|t-s|^{2 H}\right] .
$$

(5) The incremental process has a $1 / f^{\beta}$ spectrum.

Defining an incremental process by (40) and choosing $s=t-T$ :

$$
d_{H}(t)=v_{H}(t)-v_{H}(t-T)
$$

has an autocorrelation function given by

$$
R_{d}(\tau)=\frac{V_{H}}{2}\left[|\tau+T|^{2 H}+|\tau-T|^{2 H}-2|\tau|^{2 H}\right]
$$

and, as [11]

$$
F T\left[\frac{1}{2 \Gamma(\beta) \cos (\beta \pi / 2)}|t|^{\beta-1}\right]=\frac{1}{|\omega|^{\beta}}
$$

we obtain the spectrum of the incremental process:

$$
S_{d}(\omega)=\sigma^{2} \cdot \frac{\sin ^{2}(\omega T / 2)}{|\omega|^{2 H+1}} .
$$

For $|\omega| \ll \pi / T$, the spectrum can be approximated by:

$$
S_{d}(\omega) \approx \frac{\sigma^{2} T^{2}}{4} \frac{1}{|\omega|^{2 H-1}} .
$$


We conclude that the proposed definition agrees with Mandelbrot and Van Ness results.

The result expressed in (47) is interesting [1,2]:

- If $0<H<1 / 2$, the spectrum is parabolic and corresponds to an antipersistent $\mathrm{fBm}$, because the increments tend to have opposite signs; this case corresponds to the integration of a stationary fractional noise.

- If $1 / 2<H<1$, the spectrum has a hyperbolic character and corresponds to a persistent fBm, because the increments tend to have the same sign; this case corresponds to the integration of a nonstationary fractional noise.

\subsection{On the simulation of $f B m$}

The simulation of the $\mathrm{fBm}$ is a very interesting topic deserving a great deal of attention in literature. In [17] there is an exhaustive study of the simulation and identification methods of $\mathrm{fBm}$. Here we will consider the simulation task from the above formalism. We begin by obtaining the discrete-time approximation to $v_{\alpha}(t)$ in (35), using the rectangular approach, by simplicity:

$$
\hat{v}_{\alpha}(n T)=\sum_{k=0}^{n} r_{\alpha}(k T) T
$$

where $T$ is the sampling interval, that we will assume to be equal to 1 . Here, $r_{\alpha}(n)$ is a discrete-time fractional noise obtained by sampling $r_{\alpha}(t)$ in (29). Its autocorrelation function is given by (31). Its computation is a difficult task due to the somehow strange impulse response of the differintegrator \{see Eqs. (8), (9), (14), and (15)\}. Discrete-time versions can be obtained from (5), (6), (10), and (11), by fixing $h$ (normally, equal to 1). This corresponds to replacing a fractional difference for a fractional derivative leading to a discrete-time convolution that states a filtering operation with an IIR system. In the forward case it is given by [14]:

$$
\hat{r}_{\alpha}(n)=\sum_{k=0}^{\infty}(-1)^{k}\left(\begin{array}{l}
\alpha \\
k
\end{array}\right) w(n-k) .
$$

This is an $\mathrm{MA}(\infty)$ system that has an impulse response that decreases very slowly and that does not have an exact pole-zero representation:

$$
h_{n}=(-1)^{n}\left(\begin{array}{l}
\alpha \\
n
\end{array}\right) u_{n}
$$

where $u_{n}$ is the discrete-time unit step. This slowly decreasing characteristic implies that we must use a very large set of initial values to compute the output-in the simulations, we used 1000. Several attempts to overcome the problem have been considered by using ARMA models [18-21]. However, a correct evaluation and comparison of those methods remains to be done-in our simulations, we used an $\operatorname{ARMA}(6,6)$ system obtained with the algorithm described in [20]. On the other hand, the correct autocorrelation function is no longer given neither by (31), nor by (44) but by [14]:

$$
R_{r}^{\alpha}(k)=\sigma^{2} \Gamma(2 \alpha+1) \sum_{k=-\infty}^{+\infty} \frac{(-1)^{k}}{\Gamma(\alpha-k+1) \Gamma(\alpha+k+1)}
$$

To have an idea of the difference in substituting the derivative for the difference, we generate 5000 points of fractional noise by computing the convolution of a truncated impulse response obtained from (49) with white Gaussian noise. The corresponding estimated autocorrelation function is represented in the first picture in the second line. We present also the result obtained with the above referred ARMA model in the second picture of the second line. In the top pictures of each figure we present the autocorrelation functions obtained with (44) and (51). We used $H=0.3$ in Fig. 3 and $H=0.7$ in Fig. 4.

As expected, the estimated autocorrelations, although similar to the second in the first lines, are slightly different from the first. We should remark that we did not find any meaningful difference between the autocorrelations in the second line. This means that the ARMA model performs like the long MA system.

The formulation we proposed here is more general in the sense of giving the possibility of using other derivatives, specially the Grünwald-Letnikov like central derivatives [10,11]. Combining Eqs. (48) and (49) and permuting the two summations we obtain the following approximation of $\mathrm{fBm}$ :

$$
\hat{v}_{H}(n)=\sum_{m=0}^{\infty}(-1)^{m}\left(\begin{array}{c}
-H+1 / 2 \\
m
\end{array}\right) \sum_{j=1-m}^{n-m} w(j) .
$$



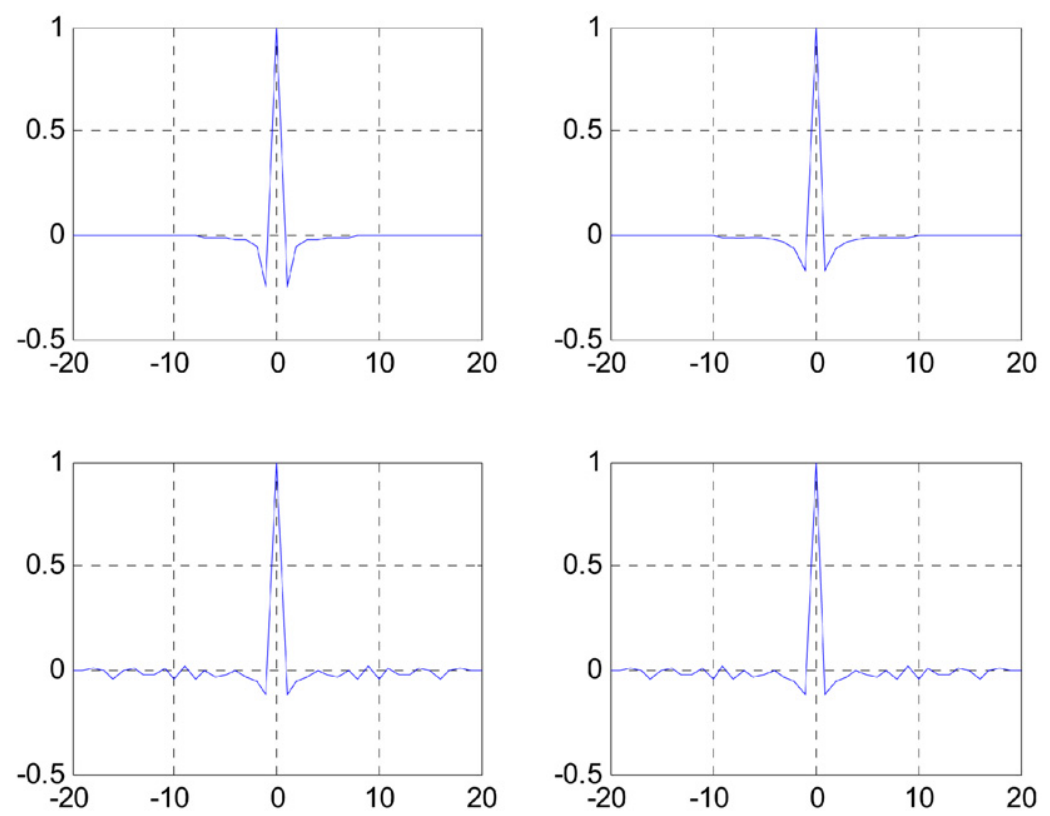

Fig. 3. Autocorrelation functions obtained, from top left to down right, with (44), (50), MA( $\infty)$, and $\operatorname{ARMA}(6,6)$ for $H=0.3$.
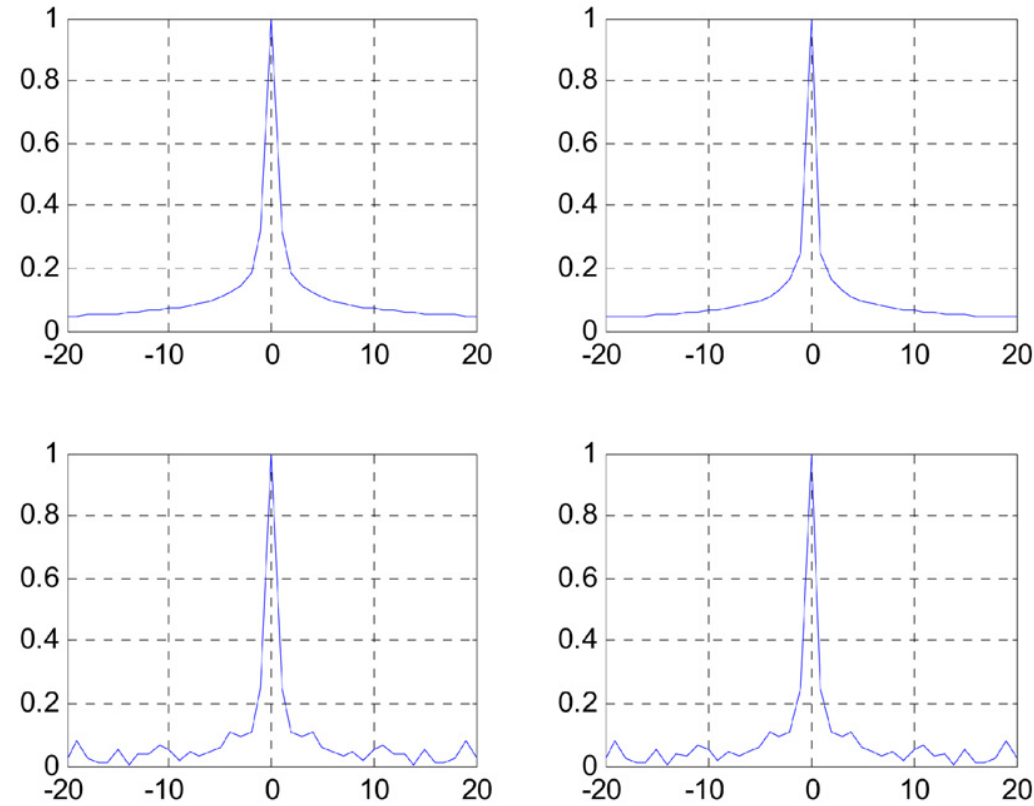

Fig. 4. Autocorrelation functions obtained, from top left to down right, with (44), (50), MA( $\infty)$, and $\operatorname{ARMA}(6,6)$ for $H=0.7$.

A close look into this formula allows us to conclude that it is a discrete-time approximation to (1). This approximation can become more accurate through the use of a trapezoidal rule in the computation of the integral. So, instead of (48), we use

$$
\hat{v}_{\alpha}(n)=\sum_{k=1}^{n} \frac{\hat{r}_{\alpha}(k-1)+\hat{r}_{\alpha}(k)}{2}
$$

that leads to:

$$
\hat{v}_{H}(n)=\sum_{m=0}^{\infty}(-1)^{m}\left(\begin{array}{c}
-H+1 / 2 \\
m
\end{array}\right) \sum_{j=1-m}^{n-m} \frac{w(j-1)+w(j)}{2}
$$

that allows us to generate a $\mathrm{fBm}$ in two steps:

(a) Smooth the white noise by adding the values inside a running $n$th length window;

(b) Compute the Grünwald-Letnikov derivative of the smoothed noise. 


\section{Conclusions}

In this Letter, we deduced a new relation between the fractional Brownian motion and the fractional derivatives of white noise. We made a brief approach into the definitions of fractional derivatives suitable for stationary stochastic processes. We considered two sets of fractional derivatives: (a) the forward and backward and (b) the central derivatives. For both sets we presented two formulations: (a) Grünwald-Letnikov and similar, and (b) integral. We showed that for these derivatives the corresponding autocorrelation functions have the same representations. The results we obtained were used to define a fractional noise and, from it, the fractional Brownian motion. We presented its properties. This gives rise to new ways of simulating the fractional Brownian motion, mainly with the use of the Grünwald-Letnikov and similar derivatives. We performed a brief study of this subject where we made a test to an ARMA approximation to the fractional derivative for generating a fractional noise.

\section{References}

[1] B.B. Mandelbrot, J.W. Van Ness, SIAM Rev. 10 (4) (1968) 422.

[2] B.B. Mandelbrot, The Fractal Geometry of Nature, W.H. Freeman and Company, New York, 1983.

[3] M.S. Keshner, Proc. IEEE 70 (1982) 212.

[4] I.S. Reed, P.C. Lee, T.K. Truong, IEEE Trans. Inform. Theory 41 (5) (1995) 1439.

[5] N.A. Kolmogorov, Wienersche Spiralen und einige andere interessante Kurven im Hilbertschen Raum, C. R. Acad. Sci. URSS 26 (2) (1940) 115.

[6] M.D. Ortigueira, A.G. Batista, Nonlinear Dynam. 38 (2004) 295.

[7] M.D. Ortigueira, A.G. Batista, On the fractional derivative of stationary stochastic processes, in: Proceedings of the Conference ECT2006, Special Session Fractional Differential Equations: Theory and Applications, Las Palmas, Spain, September 2006.

[8] M.D. Ortigueira, Fract. Calc. Appl. Signals Systems 10 (2006) 2505.

[9] M.D. Ortigueira, F.V.C. Coito, Fract. Calc. Appl. Anal. 7 (4) (2004).

[10] M.D. Ortigueira, Fractional centred differences and derivatives, in: Proceedings of the 2nd IFAC Workshop on Fractional Differentiation and Its Applications, Porto, Portugal, July 2006.

[11] M.D. Ortigueira, Int. J. Math. Math. Sci. 2006 (2006), Article ID 48391.

[12] I. Podlubny, Fractional Differential Equations, Academic Press, San Diego, 1999.

[13] S.G. Samko, A.A. Kilbas, O.I. Marichev, Fractional Integrals and Derivatives - Theory and Applications, Gordon and Breach Science, Amsterdam, 1993.

[14] M.D. Ortigueira, IEE Proc. Vision Image Signal Process. 1 (2000) 71.

[15] A.H. Zemanian, Distribution Theory and Transform Analysis, Dover, New York, 1987.

[16] G.E. Andrews, R. Askey, R. Roy, Special Functions, Cambridge Univ. Press, 1999.

[17] J.F. Coeurjolly, J. Stat. Software 5 (2000) 1.

[18] B.M. Vinagre, I. Podlubny, A. Hernandez, V. Feliu, Fract. Calc. Appl. Anal. 3 (3) (2000) 231.

[19] J.A. Tenreiro Machado, Fract. Calc. Appl. Anal. J. 1 (2001) 47.

[20] M.D. Ortigueira, A.J. Serralheiro, Fract. Calc. Appl. Signals Systems 10 (2006) 2582.

[21] M.D. Ortigueira, A.J. Serralheiro, IET Control Theory Appl. 1 (1) (2007) 173. 